Research Paper

\title{
Risk Factors for Invasive Pulmonary Aspergillosis and Hospital Mortality in Acute-On-Chronic Liver Failure Patients: A Retrospective-Cohort Study
}

\author{
Jiajia Chen, Qing Yang, Jianrong Huang, Lanjuan $\mathrm{Li}^{1,2, 凶}$ \\ 1. State Key Laboratory for Diagnosis and Treatment of Infectious Diseases, First Affiliated Hospital, College of Medicine, Zhejiang Uni- \\ versity, Hangzhou, China \\ 2. Collaborative Innovation Center for Diagnosis and Treatment of Infectious Diseases, Hangzhou, China
}

\begin{abstract}
$\triangle$ Corresponding author: Lanjuan Li. Tel: (86-571) 8723 6741. Fax: (86-571) 8723 6755. Email: ljli@zju.edu.cn
(c) Ivyspring International Publisher. This is an open-access article distributed under the terms of the Creative Commons License (http://creativecommons.org/
\end{abstract} licenses/by-nc-nd/3.0/). Reproduction is permitted for personal, noncommercial use, provided that the article is in whole, unmodified, and properly cited.

Received: 2013.01.03; Accepted: 2013.08.1I; Published: 2013.09.18

\begin{abstract}
Background: Invasive pulmonary aspergillosis (IPA) in acute-on-chronic liver failure (ACLF) patients is associated with a high mortality. But the clinical characteristics of and the risk factors for IPA among patients with ACLF remains unclear. This study was aimed at assessing clinical manifestation, the risk factors and antifungal medications for as well as the mortality due to IPA in ACLF patients at the First Affiliated Hospital, College of Medicine, Zhejiang University.

Patients and Methods: Patients with ACLF who were diagnosed with proven or probable IPA by clinical and laboratory parameters from I December 2008 to I May 2012 were retrospectively evaluated to determine the risk factors for IPA and the clinical outcomes. The follow-up ended on 30 July 2012 . Multivariate analysis was performed to identify the risk factors for mortality and the development of IPA.

Results: In total, 787 patients with ACLF were enrolled, and 39 of these patients developed IPA. Thirty seven of these 39 patients died in spite of treatment with antifungal drugs. Controls included 48 patients who did not have a pulmonary infection. The survival rate of patients with IPA was significantly lower than that of those without IPA. IPA was found to be independently associated with age $(p=0.021)$, encephalopathy $(p=0.002)$, and steroid use $(p=0.000)$. There was significant difference in the prognosis between the patients treated with either voriconazole or itraconazole and those without antifungal treatment.

Conclusions: Patients with ACLF and IPA have a high mortality rate. Patients with ACLF who present with encephalopathy should avoid steroids, as they increase the mortality rate. Azoles may prolong the survival time.
\end{abstract}

Key words: Liver failure; Invasive pulmonary aspergillosis; Antifungal agents

\section{Introduction}

Opportunistic invasive fungal infections are a major cause of morbidity and mortality in immunocompromised patients. The clinical spectrum of patients who are at risk for invasive pulmonary aspergillosis (IPA) is broad. Patients receiving allogeneic bone marrow transplant, and who have neutropenia and haematological malignancy have a high risk for
IPA. Further, patients receiving autologous bone marrow transplant, those with malnutrition, those receiving corticosteroids, HIV patients, those receiving solid organ transplant, diabetes patients, patients with underlying pulmonary diseases, and those with solid organ cancer have an intermediate risk for IPA, whereas patients with cystic fibrosis or connective 
tissue disease have a low risk for IPA[1-3]. IPA is a devastating infectious disease in intensive care unit (ICU) patients, as well as in those without any apparent predisposing immunodeficiency[4-6]. Patients with severe liver disease are at a heightened risk for infections. Acute-on-chronic liver failure (ACLF) is a life-threatening clinical syndrome with a complicated etiology, varied manifestations and a short-term mortality rate. Liver transplant seems to be an effective option to prolong survival in these group of patients, although it still can be followed by serious complications. Some studies have focused on IPA happening after liver transplantation[3,7]. It was reported invasive fungal infections (IFI) was related to high rates of morbidity and mortality in these group of patients and invasive aspergillosis tended to occur earlier in the post-transplant period[3]. But few studies have focused on Aspergillus infections in such groups of patients during pre-transplant period. They either researched the patients with HBV-related liver failure or those with hepatic failure without definite type[8,9]. The risk factors for and the clinical characteristics of IPA among patients with acute chronic liver failure (ACLF) remains unclear.

\section{Objectives}

In this retrospective-cohort study conducted over a 41-month period, was aimed at analysing the clinical characteristics of and the risk factors for the development of IPA in ACLF patients at our institution. We also focused on the survival rate of ACLF patients with IPA and the effects of different kinds of antifungal treatments on survival.

\section{Patients and Methods}

\section{Patients}

The liver disease databases of the hospital were reviewed, and consecutive patients diagnosed with ACLF, with and without IPA, were included in this retrospective study. Eligible patients had attended the First Affiliated Hospital, School of Medicine, Zhejiang University from 1 December 2008 to 1 May 2012. The follow-up continued until the date of death, date of last hospital visit, or the end of the study (30 July 2012). The minimum follow-up period for enrolled patients was 3 months after discharge. The study protocol conforms to the ethical guidelines of the 1975 Declaration of Helsinki as reflected in a priori approval by The Human Ethics Committee of the First Affiliated Hospital, School of Medicine, Zhejiang University. Informed consent was obtained from each patient included in the study. All patients received treatment in the intensive care unit (ICU) for the prevention and treatment of complications of ACLF as well as etiological treatment based on the cause of ACLF. Other conventional treatments including energy supplements were provided for the maintenance of electrolyte and acid base equilibriums.

\section{Enrolment criteria}

ACLF was defined according to following criteria specified by the Asian Pacific Association for the Study of the Liver (2009)[10]and Guideline for Diagnosis and Treatment of Liver Failure in China (2006)[11]: (1) the acute deterioration of pre-existing chronic liver disease; (2) extreme fatigue with severe digestive symptoms such as obvious anorexia, abdominal distension, or nausea and vomiting; (3) progressively worsening jaundice within a short period (serum total bilirubin $\geq 10 \mathrm{mg} / \mathrm{dL}$ or a daily elevation of $\geq 1 \mathrm{mg} / \mathrm{dL}$ ); and (4) an obvious haemorrhagic tendency with prothrombin activity (PTA) $\leq 40 \%$. The absence of any of the above 4 criteria precluded a diagnosis of ACLF.

We used the EORTC/MSG consensus definition of probable invasive Aspergillus infection, which was diagnosed if the following 2 criteria were fulfilled: 1 microbiologic criterion (positive sputum culture); and 1 major clinical criterion or 2 of minor clinical criteria (computed tomography signs suggesting lower respiratory tract infection. Proven IPA was diagnosed if fungal infection in the tissue was established by biopsy or a needle aspirate. In our study, a diagnosis of probable IPA was made on the basis of the following: (1) sputum culture positive for Aspergillus spp.; (2) bilateral nodular infiltrates with central cavitation, bilateral alveolar infiltrates, or homogeneous infiltrates in the right upper lobe; (3) the halo or air-crescent sign (a macronodule surrounded by a perimeter of ground-glass opacity) on the CT image; and (4) at least 2 minor clinical findings (signs of lower respiratory tract infection, pleural rub, and presence of any new infiltrate in a patient who did not fulfil the major criteria, but for whom an alternative diagnosis was unavailable). In brief, a diagnosis of probable IPA was made on the basis of the appearance of pulmonary consolidation or infiltrate on the thoracic image and rapid progression with antibiotic-resistant fever in the host as well as a sputum culture positive for Aspergillus spp.

ACLF patients whose chest radiograph or CT scans did not show infiltrates in the lung were included as controls.

\section{Risk factors for IPA and mortality}

The following 9 variables were assessed as risk factors for IPA: sex, age, smoking history, diabetes mellitus type 2, chronic bronchitis, steroid use, antibiotics type, encephalopathy, and neutropenia. The 
survival time for patients with ACLF was defined as the period from diagnosis of liver failure to death or the end of the study (30 July 2012). Patients who remained alive until the end of the study were censored. Survival time for ACLF patients with IPA was defined as the period from the diagnosis of IPA to death or the end of the study. Overall mortality included all deaths that occurred before the last investigation. IPA attributable mortality means the death was not clearly attributed to another cause except IPA.

\section{Statistical analysis}

Continuous variables were compared using the Mann-Whitney test, and categorical data were compared using the chi-squared test. P-values $<0.05$ were considered statistically significant for all analyses. Logistic regression was performed to identify risk factors associated with IPA in ACLF patients. A significant relationship with IPA was used as the criterion for including a variable in the multivariate stepwise modelling procedure. In the stepwise procedure, to determine the independent risk factors, we used a 0.05 significance level for entering and a 0.10 significance level for removing explanatory variables. Mortality rates among ACLF patients, with and without IPA, were plotted using the Kaplan-Meier method and compared by log-rank analysis. Survival among ACLF patients with IPA stratified by the drugs used for treatment was compared using Kruskal-Wallis analysis. A software program (SPSS16.0) was used for the statistical analyses.

\section{Results}

We identified 787 patients who were diagnosed with ACLF from 1 December 2008 to 1 May 2012. Thirty-nine patients had IPA; 48 patients without any pulmonary infection were included in the control group. The demographic and clinical characteristics of the study patients are presented in Table 1.

The subjects included 70 men and 17 women, with a median age of 39 years (range, 24-69 years). The underlying liver disease in these patients was caused by viral infection, autoimmune hepatitis, primary biliary cirrhosis, schistosomiasis, alcoholic addiction, and/or unknown reasons. Sixty patients received antibiotics for fever or the prevention of treatment complications, and 47 patients received steroids to reduce inflammation. Only 3 patients had neutropenia.

All 39 patients with IPA had a cough with expectoration, whereas 16 of these patients had hemoptysis. All 39 patients had a fever, and 12 had an initial fever lower than $38.5^{\circ} \mathrm{C}$. Among the patients with IPA, 36 presented with at least 1 macronodule with central cavitation on the chest CT scan, and 30 of these patients had multiple macronodules. Thirty-seven patients presented with infiltrates in 1 or both lungs. Twenty-eight patients had at least 1 halo or air-crescent sign.

Intravenous antifungal drugs such as caspofungin, itraconazole, or voriconazole were administered to patients diagnosed with IPA. However, some patients were not treated with antifungal therapy because they were discharged from hospital or died before the culture results were available. Twenty-four patients died due to respiratory failure in spite of treatment with antifungal drugs.

Table I. Demographic and clinical characteristics of patients with acute-on-chronic liver failure enrolled in the study, who were diagnosed with invasive pulmonary aspergillosis $(n=39)$ or without pulmonary infection $(n=48)$.

\begin{tabular}{|c|c|c|c|}
\hline Baseline characteristics & $\begin{array}{l}\text { Patients } \\
\text { without } \\
\text { pulmonary } \\
\text { infection(\%) } \\
(\mathrm{n}=48)\end{array}$ & $\begin{array}{l}\text { Patients } \\
\text { with inva- } \\
\text { sive pulmo- } \\
\text { nary asper- } \\
\text { gillosis(\%) } \\
(\mathrm{n}=39)\end{array}$ & $\begin{array}{l}p \text { val- } \\
\text { ue }\end{array}$ \\
\hline Sex (male/female) & $41 / 7$ & $29 / 10$ & 0.196 \\
\hline Age (mean \pm sd) (years) & $37.5 \pm 9.16$ & $43 \pm 11.65$ & $0.003^{*}$ \\
\hline Smoking history & $13(27.1)$ & $15(38.5)$ & 0.259 \\
\hline \multicolumn{4}{|l|}{ Underlying liver disease } \\
\hline hepatitis B virus infection & $44(91.7)$ & $37(94.9)$ & 0.560 \\
\hline hepatitis E virus infection & $1(2.1)$ & 0 & 0.367 \\
\hline primary biliary cirrhosis & 0 & $1(2.6)$ & 0.267 \\
\hline autoimmune hepatitis & $1(2.1)$ & $2(5.1)$ & 0.442 \\
\hline schistosomiasis & 0 & $1(2.6)$ & 0.115 \\
\hline acute fatty liver of pregnancy & $1(2.1)$ & 0 & 0.367 \\
\hline alcohol addiction & $3(6.3)$ & $3(7.7)$ & 0.793 \\
\hline unknown reason & $1(2.1)$ & 0 & 0.367 \\
\hline \multicolumn{4}{|l|}{ Other underlying disease } \\
\hline cardiovascular disease & $2(4.2)$ & $6(15.4)$ & 0.073 \\
\hline diabetes mellitus type 2 & 0 & $2(5.1)$ & 0.115 \\
\hline Chronic bronchitis & $1(2.1)$ & $4(10.2)$ & 0.173 \\
\hline \multicolumn{4}{|l|}{ Complications } \\
\hline hepatic encephalopathy & $4(8.3)$ & $24(61.5)$ & $0.000^{*}$ \\
\hline upper gastric bleeding & $1(2.1)$ & $1(2.6)$ & 0.882 \\
\hline hepatic renal syndrome & 0 & $11(28.2)$ & $0.000^{*}$ \\
\hline ascites & $30(62.5)$ & $32(82)$ & 0.046 \\
\hline Neutropenia & $2(4.2)$ & $1(2.6)$ & 0.831 \\
\hline $\begin{array}{l}\text { Broad-spectrum } \\
\text { antibiotics usage }\end{array}$ & $25(52.1)$ & $35(89.7)$ & $0.006^{*}$ \\
\hline One antibiotic & $15(31.2)$ & $16(41)$ & 0.347 \\
\hline Two antibiotics & $6(15.4)$ & $12(30.8)$ & 0.038 \\
\hline More than 2 antibiotics & $4(8.3)$ & $7(17.9)$ & 0.182 \\
\hline Steroid use & $12(25)$ & $35(89.7)$ & $0.000^{*}$ \\
\hline
\end{tabular}

" $p<0.01$

\section{Risk factors for IPA and all-causes mortality}

In our retrospective analysis, multivariate logistic-regression analysis showed that age $(p=0.021)$, 
hepatic encephalopathy $(p=0.001)$, and steroid use ( $p$ $=0.000$ ) were independent risk factors associated with IPA (Table 2). Furthermore, in patients who died after fungal infection, the mean time from the diagnosis of IPA to death was 4.2 (1-26) days. The all-causes mortality rate of ACLF patients with IPA was $94.87 \%$ (37 of 39 patients). IPA attributable mortality was $61.54 \%$ $(24 / 39)$. HE caused mortality was $28.2 \%(11 / 39)$. HRS attributable mortality was $5.13 \%(2 / 39)$.

All 48 ACLF patients without IPA were alive until the end of follow-up (30 July 2012). The survival rate of IPA patients was significantly lower than that of those without IPA (Figure 1).

Table 2. Logistic regression analysis of risk factors associated with invasive pulmonary aspergillosis among patients with acute-on-chronic liver failure.

\begin{tabular}{llllll}
\hline & B & $p$ value & HR & \multicolumn{2}{c}{ 95\% C.I. for HR } \\
\cline { 5 - 6 } & & & & Lower & Upper \\
\hline Age & 0.711 & 0.021 & 2.036 & 1.111 & 3.730 \\
$\begin{array}{l}\text { Hepatic encephalo- } \\
\text { pathy }\end{array}$ & 2.348 & 0.001 & 10.464 & 2.471 & 44.321 \\
Steroid usage & 2.914 & 0.000 & 18.437 & 4.352 & 78.107 \\
\hline
\end{tabular}

B: partial regression coefficient; HR: hazard ratio; CI: confident interval.

\section{Survival Functions}

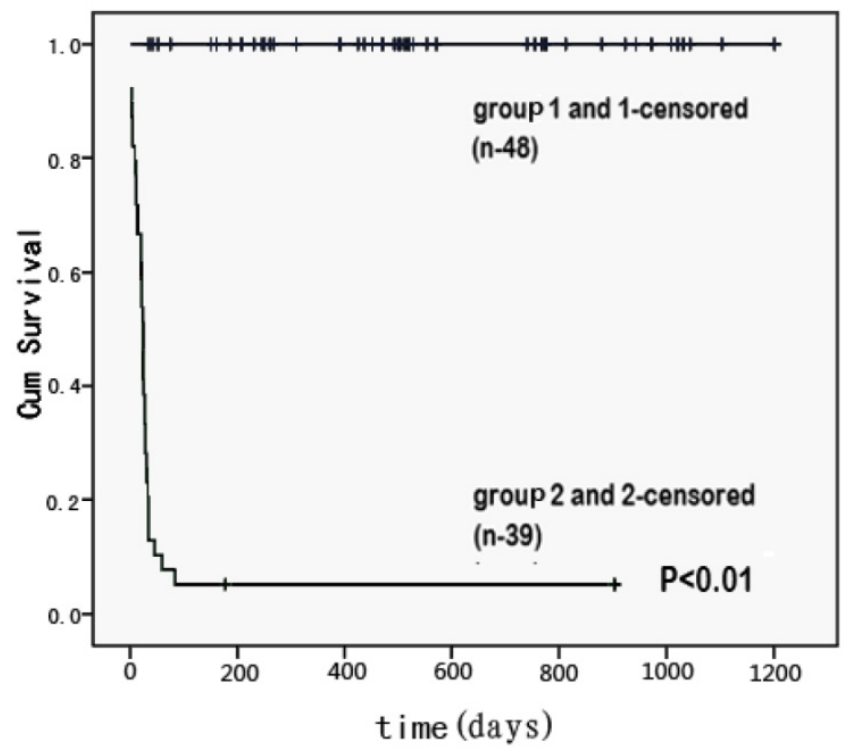

Figure I. Cumulative survival time (days) for patients with invasive pulmonary aspergillosis (group 2 and 2-censored) and without lung infection (group I and I-censored) among patients with acute-on-chronic liver failure. It represented the all causes mortality. Patients who remained alive until the end of the study were censored with minimal follow-up period of three months. There was a significant difference in the survival rate between two groups $(p<0.0 \mathrm{I})$.

\section{Influence of drug use on the survival time and IPA attributable death rate of ACLF patients with IPA}

Eight of the 10 patients taking caspofungin died within 7 days with median time of antifungal therapy of 2 days (range from 1 to 890 days). Five of the 9 patients taking voriconazole died within 7 days with median time of 6 days (range from 1 to 148 days). Four of the 7 patients taking itraconazole died within 7 days with median time of 2 days (range from 2 to 6 days). The rest 13 patients did not receive any antifungal drugs and the median survival time was 2 days (range from 1 to 6 days). Totally twenty four of 39 patients were dead with progression of fungal infection. Thirteen of 39 patients had partial response but were dead with progression of liver failure. The rest patients (2/39) got complete response and recovered after being administered either voriconazole or caspofungin. The patient was treated with voriconazole for 73 days till the infectious lesion in lung was completely absorbed on the CT image and had been alived for 148 days from diagnosis to the end of study. The patient with caspofungin had been alived for 890 days with the duration of the initial antifungal therapy for 42 days. Kruskal-Wallis analysis showed there was a significant difference in the survival time of patients within three different drugs $(p=0.011)$. Further analysis showed there was a significant difference between the survival time of patients who were administered itraconazole $(p=0.003)$ or voriconazole $(p=$ 0.015 ) and those who were not administered antifungals without consideration of the duration of antifungal therapy.

Because of the severity of ACLF, 15 patients received drugs for less than 5 days before death and were dead due to infections or other complications (Table 3). Only 11 patients got the antifungal therapy for longer than 5 days, but $45.4 \%$ patients (5/11) still were dead because of respiratory failure due to IPA. If patients who had received less than 5 days of drug were considered as those "not treated", the IPA attributable death rate was $67.8 \%(19 / 28)$. But there was no difference in IPA attributable death rate between these two groups $(p=0.691)$. There were also no differences in the constituent ratio of death among patients with different drugs and those not treated $(p=$ $0.339)$. 
Table 3. The characteristics of ACLF patients with IPA treated with or without anti-fungal drugs.

\begin{tabular}{|c|c|c|c|c|c|}
\hline Characteristics & $\begin{array}{l}\text { Caspofungin } \\
(\%)(\mathrm{n}=10)\end{array}$ & $\begin{array}{l}\text { Voriconazole }(\%) \\
(\mathrm{n}=9)\end{array}$ & $\begin{array}{l}\text { Itraconazole }(\%) \\
(\mathrm{n}=7)\end{array}$ & $\begin{array}{l}\text { Without antifungal } \\
\text { therapy }(\%)(n=13)\end{array}$ & $p$ value \\
\hline Sex(male/female) & $9 / 1$ & $6 / 3$ & $4 / 3$ & $10 / 3$ & 0.468 \\
\hline Age (mean \pm sd) (years) & $39.9 \pm 11.1$ & $51.0 \pm 12.2$ & $43.8 \pm 9.7$ & $45.8 \pm 11.9$ & 0.221 \\
\hline $\begin{array}{l}\text { Survival time } \\
(\text { mean } \pm \mathrm{sd})(\text { days })\end{array}$ & $92 \pm 280$ & $23 \pm 47$ & $6 \pm 5$ & $2 \pm 1$ & 0.011 \\
\hline \multicolumn{6}{|c|}{ Duration of antifungal therapy $\geq 5$ days } \\
\hline \multicolumn{6}{|l|}{ Reasons for death } \\
\hline Respiratory failure & $1(10.0)$ & $2(22.2)$ & $2(28.6)$ & & \\
\hline Hepaitc encephalopathy & $1(10.0)$ & $2(22.2)$ & 0 & & \\
\hline Hepatic renal syndrome & 0 & 0 & $1(14.3)$ & & \\
\hline \multicolumn{6}{|c|}{ Duration of antifungal therapy $<5$ days } \\
\hline \multicolumn{6}{|l|}{ Reasons for death } \\
\hline Respiratory failure & $5(50.0)$ & $4(44.8)$ & $2(28.6)$ & & \\
\hline Hepaitc encephalopathy & $1(10.0)$ & 0 & $2(28.6)$ & & \\
\hline Hepatic renal syndrome & $1(10.0)$ & 0 & 0 & & \\
\hline
\end{tabular}

\section{Discussion}

Fungal infections are observed in patients with various diseases, especially among immunodeficient patients such as those who receive stem cell transplantation. Although IPA has been reported in patients after liver transplantation[3,7,12], IPA has rarely been observed in patients with other liver diseases. Early diagnosis of IPA is difficult; therefore, research has focused on the identification of patients at a high risk for IPA so that antifungal prophylaxis and empirical treatment improves their survival. The patients in our study did not belong to any of the high-risk groups (patients with neutropenia, those receiving bone marrow transplant, or patients with hematological malignancy). Initially, patients with cancer, irrespective of whether or not they received hematopoietic stem cell transplantation, were considered to have a high risk for IPA, as per the Consensus guidelines (2004)[13]. In 2009, consensus guidelines revised the definition to include immunocompromised patients[14], but not critically ill patients such as ACLF patients. In our study, IPA incidence was about $5 \%$, which is as high as some hematological malignancies. There may be some similarities between these two different diseases. The first lines of host defense to aspergillus is respiratory epithelium which may be damaged due to chemotherapy or infection[15]. Then the secretion of cytokines, chemokines by the macrophages plays an important role in the coordination of innate and adaptive cellular responses to aspergillus infection[16] The successive lines of cellular defense are polymorphonuclear neutrophils and mono- cytes[17,18]. Prolonged neutropenia after chemotherapy has been reported to be associated with a poor prognosis in patients with hematological malignancies[19]. Researches showed there was a possibility of qualitative defects (impaired phagocytosis and killing of pathogens) of circulating neutrophils and granulocyte colony-stimulating factor can improve function of neutrophils from patients with acute liver failure[20,21]. The last host defense for developing invasive aspergillus infection is T-lymphocyte helper (Th) cells which exhibit successful selection and clonal expansion of direct professional effector cells with activity against Aspergillus[22,23]. Dysregulation of the host adaptive Th1/Th2 polarization may play a key role in the subacute progression of invasive aspergillosis not only in hematological malignancies but liver failure[24,25].

Reduced cellular immune function might contribute to the increased infectious morbidity of these patients with ACLF. Patients diagnosed with probable IPA may, in fact, have a proven infection. If the tissue is positive for fungal infections, the host factors can be omitted. However, the diagnosis of proven IPA is difficult because of coagulation disorder of the patients with ACLF. Therefore, it is unfeasible to obtain the infectious tissue through invasive procedures. In our study, only 1 patient had evidence of fungal infection in the tissue (data not shown).

Hence, we analysed the relationship of 9 variables with the development of IPA. The previous study showed multiple antibiotics use and frequent invasive procedures were independent factors associated with the occurrence of IPA in patients with HBV-related 
liver failure[8]. And the patients were prone to many types of infections caused by opportunistic pathogens including Aspergillus spp. when using multiple antibiotics. However in our study results showed there was no significant difference in antibiotics use between the patients with IPA and those without lung infection. Instead, age, encephalopathy, and steroid use were significant risk factors for IPA. All 3 factors indirectly reflect the immune status. Encephalopathy is known to be closely related to the high mortality of liver failure. Moreover, as we know Aspergillus can be detected throughout the environment. For patients in a coma, encephalopathy increases the chance of aspiration which may induce infection. Although not all the patients used steroids, mortality was much higher in those who were given steroids. Corticosteroids influence phagocyte function by the impairment of phagocytosis, oxidative burst, cytokine and chemokine production, and cellular migration[26]. Several studies have demonstrated that corticosteroids impair the functional ability of phagocytes to kill A. fumigatus conidia and hyphae[27-29], and it has been listed as a host factor in the diagnosis of IPA.

Aspergillus fumigatus, which was found to be sensitive to azole, was the most common species identified in our study (data not shown in result). It is the primary causative agent of fungal infections in humans, followed by A. flavus, A. terreus, and A. Niger $[2,30]$. In our study, more than half of ACLF patients with IPA died due to respiratory failure despite antifungal therapy. Although voriconazole and itraconazole may prolong the life time of ACLF patients with IPA, there were no differences in IPA attributable death rate and another reasons attributable death rate among the patients with or without drugs. Moreover most of patients had not enough time to be treated with antifungal drugs and dead with IPA induced respiratory failure. So we took this into consideration and found there was also no difference in the constituent ratio of death among patients with different drugs and those not treated including those without enough duration. IPA attributable death rate with drugs was lower than those not treated. It was suggested antifungal therapy may reduce IPA attributable death, but there was no statistically difference. Maybe the numbers of patients enrolled in the study were too limited to make a real comparison. Much more cases should be further studied. Moreover, we know that voriconazole may induce liver and kidney injuries. The dose should be adjusted according to the underlying kidney or liver diseases. But in our study the doses for the surviving patient were not changed even though he had abnormally high total bilirubin levels. Despite the poor prognosis of aspergillosis, liver and renal function did not worsen after treatment, and the patient still recovered progressively and survived till the last investigation. Maybe he was young enough to be cured by an antifungal agent.

It has been reported that IPA attributable mortality rate ranges from $17.3 \%$ to $90 \%$ in high-risk populations and depends on the host factors, site of infection, and the treatment regimen applied[31,32]. The mortality due to ACLF is still very high, although artificial liver support system and liver transplantation can reduce the mortality[33,34]. Patients treated in more recent years may have better outcomes because of new developments in diagnosis and early treatment with new antifungal drugs, but the mortality rate of ACLF due to IPA was still as high as $61 \%$ in our study, suggesting that IPA may be an important predictor of mortality in ACLF patients. There are some possible reasons: 1 . The severity of the cellular immunocompromise of ACLF which was discussed above made patients vulnerable to the infection of aspergillus. 2. In such kind of patients the coagulation disorder contributed to the hemorrhage of upper respiratory tract easily when aspergillus invased into the alveolar vessels. 3 . In our study it was late to begin the antifungal therapy although immediately after the culture result was known. Moreover, almost one-third patients did not receive the antifungal drugs. Patients may miss the best time for the treatment. So early diagnosis of IPA and an earlier treatment may improve the prognosis of these patients. Physicians unfamiliar with the clinical manifestations of IPA may not consider symptoms such as a relatively low fever with cough or chest pain. In addition, they may believe that the sputum culture was positive because of contamination and miss the opportunity for treatment. Further, some patients in our study may not have obtained a CT scan. The new Aspergillus galactomannan EIA was approved by the $\operatorname{FDA}[35,36]$. However, our hospital did not conduct the GM-test regularly, which may have resulted in substantial underdiagnosis and delay in treatment. To determine whether prophylaxis was useful in improving the prognosis, additional studies are needed with more number of patients.

In summary, patients with ACLF were immunocompromised hosts who were prone to IPA, and the mortality rate of these patients is high. The greatest risk factor for infection is use of steroids, which increases the susceptibility of the patient to IPA. What's more, ACLF patients with encephalopathy should not be treated using steroids, as they increase the mortality rate further. When a GM test cannot be performed and symptoms of pneumonia such as cough or hemoptysis in ACLF appear, IPA should be considered. Hence, once patients with ACLF are diagnosed with 
IPA, antifungal therapy should be initiated immediately. However, data on prophylaxis chemotherapy are lacking, and then, additional investigation is necessary. Although the use of voriconazole without adjusting the dose did not negatively impact liver and renal function in the current study, we should closely monitor liver and renal function effects in patients with ACLF.

\section{Acknowledgements}

Jiajia Chen, Qing Yang and Lanjuan Li participated in the design of the study, analysis and interpretation of data, and drafting of the manuscript. Jianrong Huang participated in the interpretation of data and critical review of the manuscript for important intellectual content. This work was supported by the Chinese High Tech Research \& Development (863) Program (No. 2011AA020104) and National Science and Technology Major Project (No. 2012ZX10002004).

\section{Competing Interests}

The authors have declared that no competing interest exists.

\section{References}

1. Perfect JR, Cox GM, Lee JY, et al. The impact of culture isolation of Aspergillus species: a hospital-based survey of aspergillosis. Clin Infect Dis. 2001;33:1824-33.

2. Morgan J, Wannemuehler KA, Marr KA, et al. Incidence of invasive aspergillosis following hematopoietic stem cell and solid organ transplantation: interim results of a prospective multicenter surveillance program. Med Mycol. 2005; 43(Suppl. 1): S49-S58.

3. M Zicker, AL Colombo, BH Ferraz-Neto, et al. Epidemiology of fungal infections in liver transplant recipients: a six-year study of a large Brazilian liver transplantation centre. Mem Inst Oswaldo Cruz. 2011; 106: 339-345.

4. .Vandewoude KH, Blot SI, Depuydt P, et al. Clinical relevance of Aspergillus isolation from respiratory tract samples in critically ill patients. Crit Care. 2006;10:R31.

5. Barnes AJ,Denning DW. Aspergilli-significance as pathogens. Rev Med Microbiol. 1993;4:176-180.

6. Meersseman W, Vandecasteele SJ, Wilmer A, et al. Invasive aspergillosis in critically ill patients without malignancy. Am J Respir Crit Care Med. 2004;170:621-5.

7. Pacholczyk M, Lagiewska B, Lisik W, et al. Invasive fungal infections following liver transplantation - risk factors, incidence and outcome. Ann Transplant. 2011;16:14-6.

8. Wang W, Zhao CY, Zhou JY, et al. Invasive pulmonary aspergillosis in patients with HBV-related liver failure. Eur J Clin Microbiol Infect Dis. 2011;30:661-7.

9. Li D, Chen L, Ding X, et al. Hospital-acquired invasive pulmonary aspergillosis in patients with hepatic failure. BMC Gastroenterol. 2008;8:32.

10. Sarin Shiv Kumar, Kumar Ashish, Almeida John A, et al. Acute-on-chronic liver failure: consensus recommendations of the Asian Pacific Association for the study of the liver (APASL). Hepatol Int. 2009;3:269-82.

11. Liver Failure and Artificial Liver Group, Chinese Society of Infectious Diseases, Chinese Medical Association; Severe Liver Diseases and Artificial Liver Group, Chinese Society of Hepatology, Chinese Medical Association. Diagnostic and treatment guidelines for liver failure. Zhonghua Gan Zang Bing Za Zhi. 2006;14: 643-6.

12. Takeda K, Tanaka K, Kumamoto $\mathrm{T}$, et al. Emergency versus elective living-donor liver transplantation: a comparison of a single center analysis. Surg Today. 2012;42:453-9.

13. Ascioglu S, Rex JH, de Pauw B, et al. Defining opportunistic invasive fungal infections in immunocompromised patients with cancer and hematopoietic stem cell transplants: an international consensus. Clin Infect Dis. 2002;34:7-14.

14. De Pauw B, Walsh TJ, Donnelly JP, et al. Revised definitions of invasive fungal disease from the European Organization for Research and Treatment of Cancer/Invasive Fungal Infections Cooperative Group and the National Institute of Allergy and Infectious Diseases Mycoses Study Group (EORTC/MSG) Consensus Group. Clin Infect Dis. 2008;46:1813-21.

15. Estey EH, Keating MJ, McCredie KB, et al. Causes of initial remission induction failure in acute myelogenous leukemia. Blood. 1982;60:309-15.

16. Clemons KV, Calich VL, Burger E, et al. Pathogenesis I:interactions of host cells and fungi. Med Mycol 2000;38:99-111.

17. Schaffner A. Macrophage-Aspergillus interactions. Immunol Ser 1994;60:545-52.

18. Schaffner A, Douglas H, Braude A. Selective protection against conidia by mononuclear and against mycelia by polymorphonuclear phagocytes in resistance to Aspergillus: observations on these two lines of defense in vivo and in vitro with human and mouse phagocytes. J Clin Invest.1982;69:617-31.

19. Pagano L, Caira M, Candoni A, et al. Invasive aspergillosis in patients with acute myeloid leukemia: a SEIFEM-2008 registry study. Haematologica. 2010;95:644-50.

20. Taylor NJ, Nishtala A, Manakkat Vijay GK, et al. Circulating neutrophil dysfunction in acute liver failure. Hepatology. 2013;57:1142-52

21. Rolando N, Clapperton M, Wade J, et al. Granulocyte colony-stimulating factor improves function of neutrophils from patients with acute liver failure. Eur J Gastroenterol Hepatol. 2000;12:1135-40.

22. Cenci E, Perito S, Enssle KH, et al. Th1 and Th2 cytokines in mice with invasive aspergillosis. Infect Immun. 1997;65:564-70.

23. Cenci E, Mencacci A, Fe d'Ostiani C, et al. Cytokine- and T helper-dependent lung mucosal immunity in mice with invasive pulmonary aspergillosis. J Infect Dis. 1998;178:1750-60.

24. Casadevall A. Acquired immunity against fungi. In: Ahmed R, ed. Immunology of infectious diseases. Washington, DC: American Society for Microbiology Press, 2002.

25. Wasmuth HE, Kunz D, Yagmur E, et al. Patients with acute on chronic liver failure display "sepsis-like" immune paralysis. J Hepatol. 2005;42:195-201.

26. Lionakis, MS, and DP Kontoyiannis. Glucocorticoids and invasive fungal infections. Lancet. 2003;362:1828-1838.

27. Waldorf AR, SM Levitz and RD Diamond. In vivo bronchoalveolar macrophage defense against Rhizopus oryzae and Aspergillus fumigatus. J Infect Dis. 1984;150:752-760.

28. Meier-Osusky, I, G Schoedon, F Blauer, et al. Comparison of the antimicrobial activity of deactivated human macrophages challenged with Aspergillus fumigatus and Listeria monocytogenes. J Infect Dis. 1996;174:651-654.

29. Kamberi M, E Brummer, and DA Stevens. Regulation of bronchoalveolar macrophage proinflammatory cytokine production by dexamethasone and granulocyte-macrophage colony-stimulating factor after stimulation by Aspergillus conidia or lipopolysaccharide. Cytokine.2002;19:14-20.

30. GP Bodey and S Vartivarian. Aspergillosis. Eur J Clin Microbiol Infect Dis. 1989;8:413-37.

31. Lin SJ, J Schranz and SM Teutsch. Aspergillosis case-fatality rate: systematic review of the literature. Clin Infect Dis. 2001;32:358-366.

32. AM Nosari, M Caira, ML Pioltelli, et al. Hema e-Chart Registry of invasive fungal infections in haematological patients: improved outcome in recent years in mould infections. Clin Microbiol Infect. 2013;19:757-762.

33. Qian Y, Lanjuan L, Jianrong H, et al. Study of severe hepatitis treated with a hybrid artificial liver support system. Int J Artif Organs. 2003;26:507-13.

34. Ling $Q, X u X$, Wei $Q$, et al. Downgrading MELD improves the outcomes after liver transplantation in patients with acute-on-chronic hepatitis B liver failure. PLoS One. 2012;7:e30322.

35. Tănase AD, Coliță A, Mărculescu A, et al. Using the galactomannan antigen assay in the diagnosis of invasive aspergillosis after hematopoietic stem cell transplantation. Rom J Morphol Embryol. 2012;53:379-82.

36. Wheat LJ, Walsh TJ. Diagnosis of invasive aspergillosis by galactomannan antigenemia detection using an enzyme immunoassay. Eur J Clin Microbiol Infect Dis. 2008;27:245-51. 